\title{
Differentiation of Mesenchymal Stem Cells into Photoreceptor-like Cells under the Influence of a Temporary Increase in miRNA-182, -183 Expression
}

Mohammad-Reza Mahmoudian-Sani

Shahid Chamran University of Ahvaz Faculty of Science

Najmeh Fattahi

Shahrekord University of Medical Science

Samaneh Arab

Shahrekord University of Medical Science

samira asgharzade ( $\nabla$ asgharzade2336@gmail.com )

Shahrekord University of Medical Science

\section{Research Article}

Keywords: Human Bone Marrow-Derived Mesenchymal Stem Cell, miRNA-182, miRNA-183, Photoreceptor cell

Posted Date: August 9th, 2021

DOI: https://doi.org/10.21203/rs.3.rs-748878/v1

License: (c) (i) This work is licensed under a Creative Commons Attribution 4.0 International License. Read Full License 


\section{Abstract}

It is found that the death of retinal photoreceptors is the main cause of retinal degeneration, while there is not an effective treatment protocol. Data of preclinical and clinical trials indicates that the stem cell therapy is a useful way of treating retinal degeneration problems. On the other hand, previous works found that miRNA-182, -183 significantly affected the photoreceptor maturation and maintenance in animal models. The present study aimed to investigate the impact of a temporary increase in miRNA-182, -183 expression on the differentiation of human bone marrow mesenchymal stem cells (hBMSCs) into photoreceptor-like cells. To this end, miRNA-182, -183 was transfected into hBMSCs; then, qRT-PCR was performed to measure the expression levels of miRNA-182, -183 and some retina-specific genes such as $\mathrm{OTX} 2, \mathrm{NRL}, \mathrm{PKCa}$, and recoverin. $\mathrm{CRX}$ and rhodopsin (RHO) levels were also measured through qRT-PCR and immunocytochemistry We indicated that the transfection of hBMSCs with miRNA-182, -183 using the Lipofectamine induce differentiation and progenitor's genes expression consisted of CRX, OTX2, PKC, Recoverin, NRL and RHO. Moreover, the upregulation expression of transcription factors, $\mathrm{CRX}$ and RHO, indicated that miRNA-182, -183 could serve as crucial functions in the differentiation of hBMSCs into photoreceptor-like cells. The findings may provide a new strategy to improve the usage of hBMSCs as a treatment for the retinal dysfunction.

\section{Introduction}

There are about 37 million blind people around the world; and most popular conditions are macular degeneration, diabetic retinopathy, and glaucoma ${ }^{1}$. Despite the fact that advances in laser and surgical procedure and drug discovery have decreased the vision loss due to these conditions, much of the vision loss, which is associated with degenerative retinal conditions, is still inalterable and advances over time 2,3 . In recent years, the stem cell therapy has been considered as a promising method of treatment of retinal diseases. Stem cells have the self-renewal ability and also can be developed to numerous cell types. Retinal anatomy has some advantages for stem cell therapy such as small space, local delivery of cells to the retina, and limited number of required stem cells. The ideal stem cell for cell substitution in eyes with retinal disorders is a cell that can be easily expanded and give rise to multiple cells ${ }^{4}$. Among numerous stem cell types, which have been tested for treatment of retinal diseases, the mesenchymal stem cells (MSCs) are attractive candidates as they can be easily obtained from bone marrow or adipose tissue, and be manipulated and used for autologous stem cell transplantation in clinics ${ }^{5,6}$. MicroRNAs (miRNAs), which are small-noncoding RNAs, play effective roles as key modulators of many physiological and pathophysiological processes often by acting as regulators of gene expression events 7. An important family of miRNAs, which are known as sensory organen-riched miRNA clusters, is the microRNA-183 cluster consisting of miR-183, miR-96 and miR-182. It is found that these miRs are highly expressed in adult retina and other sensory organs. Moreover, their expression is necessary and sufficient for the photoreceptor maturation and maintenance ${ }^{8,9}$. The miR-96/182/183 cluster is necessary for the maintenance of Cone Photoreceptor Outer Segments in vitro and visual function in vivo ${ }^{10}$. Therefore, key roles of miRNAs cannot be ignored in photoreceptors. In this regard, miR-183 is also known as an 
important epigenetic regulator which plays role in the physiological function and is investigated in different pathologic conditions by various studies. The miR-182 knockout rats show serious structural defects in their photoreceptors. A large amount of information has been recently obtained on pivotal functions of miRNAs in the vertebrate retina. Global and specific miRNA loss-of-function studies play key roles in the miRNA development, physiology and diseases of retina ${ }^{11-13}$. New technologies for measuring the expression of miRNA genes allow the accurate evaluation of results of removing specific miRNAs. These technologies also provide the opportunity to evaluate the interaction of miRNA with the target mRNA in a specific tissue or cell ${ }^{14}$. Various studies have found that the P347S rhodopsin transgenic mice, human retinitis pigmentosa (RP) model, and three other types of photoreceptor-linked mouse models express the miR-96/182/183 cluster. These results confirm the association between the reduction of their gene expression and retinal disease in humans ${ }^{15}$. All these studies aim to help stem cell-based therapies among a large number of people who are affected by disorders that severely challenge the quality of life of themselves and their acquaintances. The present study evaluated the differentiation of Human bone marrow-derived mesenchymal stem cells (hBMSCs) that highly expressed miRNA-182, -183 towards photoreceptor like cells.

\section{Materials And Methods}

\section{Cell culture}

Human bone marrow-derived mesenchymal stem cells (hBMSCs) were purchased from Institute Pasteur of Iran (Tehran, Iran), and cultured in the Dulbecco's Modified Eagle's Medium (DMEM, Sigma, Steinhem, Germany) containing 15\% fetal bovine serum (FBS) (Gibco, Grand Island, MA, USA) and 2 mM Lglutamine (Sigma) 1\% streptomycin and penicillin (Sigma). The medium was then replaced every three days, and the fourth or fifth passage was used for the transfection with miRNA-182, -183.

\section{Cell Transfection}

The hBMSCs transfection was carried out using Lipofectamine (Invitrogen's Lipofectamine 2000) based on company instructions. A total number of $1.5 \times 10^{4}$ cells were cultured in a 6 -well culture plate containing DMEM without FBS; and antibiotics were added to each well. Afterwards, $50 \mathrm{nM}$ of miRNA-182 and -183 with $10 \mu \mathrm{l}$ of Lipofectamine were mixed in $200 \mu \mathrm{l}$ of DMEM culture medium; and the mixture was incubated at room temperature for 15 minutes. The presented complex was added to hBMSCs; and the environment containing FBS and antibiotics were added to cells after 5 hours of incubation, and the cells were incubated for 24 and 48 hours. The transfected scrambled cells were simultaneously cultured with miRNA-182, -183 transfected cells. The evaluation of cell transfection was also carried out via the RT-PCR. Each transfection was performed in the triplicate; and the cells were $60-70 \%$ confluent by the time of the transfection.

\section{MicroRNA and RNA Extraction}


MicroRNA and RNA Extraction

MicroRNA was isolated by miRNeasy isolation kit (Qiagen) according to the manufacturer's instructions. miRCURY LNA miRNA Mimics include two short, LNA-enhanced complimentary strands which prevent any miRNA-like activity associated with passenger strands; hence, they can be sure that observed phenotypes using these mimics are due to the increased activity of the mimicked miRNA. Total RNA was extracted from cultured cells (transfected with miRNA and control cells) with TRIzol (Ambion) according to the protocol. The quality of RNA samples was then measured by NanoDrop (Thermo Fisher Scientific).

\section{Real Time PCR}

Approximately 2 micrograms of RNA were used for the synthesis of cDNA using the thermo scientificK1622 kit and rotor-gene Q (Qiagen, USA). The thermal conditions included the initial stage of denaturation at $95^{\circ} \mathrm{C}$ for 3 minutes, and then for 40 cycles at $95^{\circ} \mathrm{C}$ and 15 seconds and $72{ }^{\circ} \mathrm{C}$ for 25 seconds. Table 1 presents the applied primers for each studied gene. The mRNA relative expression level was also calculated using the $2-\Delta \Delta C t$ method. mRNA levels were normalized using the GAPDH as a housekeeping gene.

Table 1

Primers used in qRT-PCR

\begin{tabular}{|c|c|}
\hline Gene & Sequence $\left(5^{\prime}->3^{\prime}\right)$ \\
\hline \multirow[t]{2}{*}{ CRX } & GGAGCTGGAGGCACTGT \\
\hline & ACAAACCTGAACCCTGGAC \\
\hline \multirow[t]{2}{*}{ OTX2 } & CATGAGGCTGTAAGTTCCAC \\
\hline & TTGTTTGGAGGTGCAAAGTC \\
\hline \multirow[t]{2}{*}{ PKC } & GATCGCCACCTACCGCAA \\
\hline & CCTCAGGCACAGTCGTCT \\
\hline \multirow[t]{2}{*}{ Recoverin } & GGGACCATCAGCAAGAAT \\
\hline & GATCTTCTCGGCTCGCTTT \\
\hline \multirow[t]{2}{*}{$\mathrm{RHO}$} & GTCCAGGTACATCCCCG \\
\hline & ACGAACATGTAGATGACAA \\
\hline \multirow[t]{2}{*}{ SLC1A1 } & ATTCGTGTTACCCGTTGGTG \\
\hline & CCCAAGTCCAGGTCATTCAA \\
\hline \multirow[t]{2}{*}{ GAPDH } & GAGTCCACTGGCGTCTTCAC \\
\hline & ATGACGAACATGGGGGCATC \\
\hline
\end{tabular}




\section{Immunocytochemistry (ICC)}

Cells, which were seeded onto precoated chamber slides, were fixed with $4 \%$ paraformaldehyde (SigmaAldrich), permeabilized using $0.1 \%$ Triton-X100 in PBS, and blocked with 1\% BSA. The cells were then immunostained by anti-CRX and anti-RHO antibody (1:500) (Santa Cruz Biotechnology) and conjugated secondary antibodies in PBS (1:500; Santa Cruz Biotechnology). After washing, cell nuclei were counterstained with DAPI. Negative control samples were processed in parallel, but without any primary antibody. Fluorescence images were captured with a fluorescence's microscope (Olympus Corp., Tokyo, Japan).

\section{Statistical Analysis}

Results are presented as mean \pm SD. Kruskal-Wallis test and Mann-Whitney $U$ test were respectively used to compare gene expression levels in groups and also determine significance levels of groups. P-values lower than 0.05 were also considered significant. Statistical analysis and graph generation for gene and protein expression were conducted using GraphPad Prism (Version 7). Other data was analyzed using the SPSS 16.00 .

\section{Results}

\section{Expression of miRNA-182, -183}

The real time PCR was performed 24 and 48 hours after the transfection to assess the expression in hBMSCs that were transfected by miRNA-182, -183 and scramble. Our results indicated that levels of miRNA-182, -183 expression increased in miRNA-182, -183 transfected cells in both times compared with scramble transfected cells (Fig. 1).

\section{Expression of progenitor and differentiation genes}

The real time PCR was performed 24 and 48 hours after the transfection in order to examine the effects of transfection with miRNA-182, -183 on progenitor and differentiation genes that included CRX, OTX2, NRL, SLC1A1, PKC, RHO and Recoverin. Figure 2 shows that the expression of CRX, OTX2 and RHO genes is significantly upregulated after 24 hours compared with the control. CRX $\square \mathrm{RHO}$ and PKC gene expression also significantly increased after 48 hours compared with the control in transfected cells with miR182 (Figure 3).

Figure 4 shows that the expression of OTX2 gene is significantly upregulated after 24 and 48 hours compared with the control. According to the Figure 5, the RT-PCR revealed no changes in CRX, NRL, RHO, Recoverin, SLC1, and PKC mRNAs in the transfected cells (miR183) after $24 \mathrm{~h}$. Furthermore, CRX, NRL, $\mathrm{RHO}$, Recoverin and PKC gene expression significantly increased after 48 hours compared with the control (Figure 6). 


\section{Protein expression of CRX and RHO}

The expression of $\mathrm{CRX}$ and $\mathrm{RHO}$ was assessed by immunocytochemistry technique for further investigation of the impact of activity of miRNA-182, -183 on photoreceptors markers. hBMSCs were observed by a fluorescence microscope (Olympus, Tokyo, Japan) at 10x and 20x magnifications. The cell nuclei were stained with 4, 6-diamidino-2-phenylindole staining (DAPI). As shown in Figures 7, 8, 9, and 10. hBMSCs, which were transfected by miRNA-182, -183 , express both CRX and RHO markers in 24 and 48 hours after the transfection.

\section{Discussion}

The proper function of a vision system depends on functions of photoreceptor cells. Unfortunately, these cells do not self-regenerate, and eyesight decreases due to the loss of these cells depending on the extent of their destruction, and eventually the person becomes blind ${ }^{16}$. The use of stem cells, which can differentiate into different cell types, has brought a new hope in the treatment of these patients 5,17. Human adult bone marrow mesenchymal stem cells (hBMSCs) are a noteworthy choice for an ophthalmological research due to the fantastic ability such as easy expanding and broad differentiation potential; and it may also offer the possibility of autologous transplantation ${ }^{18}$. The miR-183 family is considered to be the most abundant and effective miRNA that is involved in the regulatory and evolutionary processes of the nervous system of vision because their elimination lead to the blindness ${ }^{19}$. Fan et al. indicated an essential role of the miR-183 family in driving multiple sensory receptor cell differentiation; and the loss of miR-183 Cluster leads to the sensory malfunction in Mice. ${ }^{20}$. In the present study, miRNA-182, -183 were transfected into hBMSCs and it evaluated the expression levels of miRNA-182, -183 and some photoreceptor markers (RHO, Recoverin) and Vision nerve markers (PKC, OTX2, CRX). Previous research found that CRX acted as a master transcription factor for reprogramming peripheral blood mononuclear cells (PBMCs) into photoreceptor-like cells ${ }^{21}$. Transcription factor OTX2 is necessary for the photoreceptor cell terminal differentiation and plays an essential role in the maturation of retinal photoreceptor and bipolar cells ${ }^{22}$. Rhodopsin is a photopigment that converts light into an electrical signal and is encoded by the rhodopsin (RHO) gene. In humans, mutations of $\mathrm{RHO}$ are associated with the retinitis pigmentosa ${ }^{23}$. Protein Kinase $C(P K C)$ is a type of serine/threonine kinases that are expressed in retinal progenitor cells in order to control the photoreceptor development ${ }^{24}$. Recoverin, a photoreceptor-specific calcium-binding protein, is necessary for the transduction of light by photoreceptor cells ${ }^{25}$. In the present study, the Real time PCR analysis revealed that increased expression levels of miRNA-182, -183 could lead to a higher expression of RHO, PKC, OTX2, NRL, Recoverin and CRX in vitro. In addition, Immunofluorescence results indicated a significant rise in the protein expression of CRX and RHO in the transfected group with miRNA-182, -183. Davari et al. found that the human retinal pigment epithelial (hRPE) was transfected with miR-183/-96/-182 genes in order to transdifferentiate into retinal neuron-like cells. They detected that retina-specific neuronal genes such as OTX2, NRL, PDC and DCT were upregulated in hRPE cells that were influenced by the ectopic overexpression of miR-183 
cluster. Furthermore, the protein expression of photoreceptor specific markers like rhodopsin, red opsin, $\mathrm{CRX}$ and ganglion cell marker Thy 1 in hRPE transfected cells was confirmed by the immunocytochemistry staining ${ }^{26}$. Komuta et al. transduced CRX genes into PBMCs by Sendai virus vectors. They found that numerous retinal disease-related genes were efficiently detected in CRX transduced cells; and most of them played critical roles in photoreceptor functions, but the expression of Rhodopsin was low. ${ }^{27}$ Zhang et al. found that the human embryonic stem cells (hESC) and human induced pluripotent stem cells (hiPSC) were differentiated under the treatment with B27, Dkk1, Noggin, IGF-1; and then N2 and FGF were able to significantly express retinal specific markers such as NF, Recoverin and Rhodopsin ${ }^{28}$ In general, our results first indicated that miRNA-182, -183 could help to differentiate bone marrow mesenchymal stem cells into photoreceptor cells by increasing the expression of RHO, Recoverin, PKC, NRL, OTX2, and CRX genes.

\section{Declarations}

\section{Author contribution}

Mohammad-Reza Mahmoudian-Sani, and Samira Asgharzade designed the research Mohammad-Reza Mahmoudian-Sani, Najmeh Fattahi, Samaneh Arab, Samira Asgharzade performed experiments. Samira Asgharzade and Mohammad-Reza Mahmoudian-Sani analyzed data and wrote the paper. All the authors read and approved the final manuscript.

\section{Acknowledgments :}

This study was supported by the deputy of Shahrekord University of Medical Sciences under the grant number of 2628 and 3098. The ethical code for this research is IR.SKUMS.REC.1396.200 and IR.SKUMS.REC.1398.056.

\section{Funding :}

This research was supported by the Research Deputy of Shahrekord University of Medical Sciences (grant number of 2628 and 3098).

\section{Conflict of interest:}

The authors declare that there are no conflicts of interest.

\section{Ethics approval}


This study was approved by Iran National Committee for Ethics in Shahrekord University of Medical Sciences (IR.SKUMS.REC.1396.200 and IR.SKUMS.REC.1398.056).

\section{Consent to participate:}

Informed consent was obtained from all participants of the study.

\section{Consent to publish:}

The participant was informed that the results of this research would be published in the journal.

\section{References}

1. Foster A, Resnikoff S (2005) The impact of Vision 2020 on global blindness. Eye 19(10):1133

2. Asbell PA, Dualan I, Mindel J, Brocks D, Ahmad M, Epstein S (2005) Age-related cataract. The Lancet 365(9459):599-609

3. Stitt AW, Curtis TM, Chen M et al (2016) The progress in understanding and treatment of diabetic retinopathy. Progress in retinal eye research 51:156-186

4. Park SS, Moisseiev E, Bauer G et al (2017) Advances in bone marrow stem cell therapy for retinal dysfunction. Progress in retinal eye research 56:148-165

5. Holan V, Hermankova B, Kossl J (2017) Perspectives of Stem Cell-Based Therapy for Age-Related Retinal Degenerative Diseases. Cell transplantation 26(9):1538-1541

6. Huang Y, Enzmann V, Ildstad ST (2011) Stem cell-based therapeutic applications in retinal degenerative diseases. Stem Cell Reviews Reports 7(2):434-445

7. He L, Hannon GJ (2004) MicroRNAs: small RNAs with a big role in gene regulation. Nature Reviews Genetics 5(7):522

8. Karali M, Peluso I, Marigo V, Banfi S (2007) Identification and characterization of microRNAs expressed in the mouse eye. Investigative ophthalmology visual science 48(2):509-515

9. Xu S, Witmer PD, Lumayag S, Kovacs B, Valle D (2007) MicroRNA (miRNA) transcriptome of mouse retina and identification of a sensory organ-specific miRNA cluster. Journal of Biological Chemistry 282(34):25053-25066

10. Busskamp V, Krol J, Nelidova D et al (2014) miRNAs 182 and 183 are necessary to maintain adult cone photoreceptor outer segments and visual function. Neuron 83(3):586-600

11. Lumayag S, Haldin CE, Corbett NJ et al (2013) Inactivation of the microRNA-183/96/182 cluster results in syndromic retinal degeneration. Proceedings of the National Academy of Sciences of the United States of America 110(6):E507-E516

12. Xu S, Witmer PD, Lumayag S, Kovacs B, Valle D (2007) MicroRNA (miRNA) transcriptome of mouse retina and identification of a sensory organ-specific miRNA cluster. The Journal of biological 
chemistry 282(34):25053-25066

13. Zhu Q, Sun W, Okano K et al (2011) Sponge transgenic mouse model reveals important roles for the microRNA-183 (miR-183)/96/182 cluster in postmitotic photoreceptors of the retina. The Journal of biological chemistry 286(36):31749-31760

14. Sundermeier TR, Palczewski K (2012) The physiological impact of microRNA gene regulation in the retina. Cellular molecular life sciences: CMLS 69(16):2739-2750

15. Loscher CJ, Hokamp K, Kenna PF et al (2007) Altered retinal microRNA expression profile in a mouse model of retinitis pigmentosa. Genome biology 8(11):R248

16. Scholl HP, Strauss RW, Singh MS et al (2016) Emerging therapies for inherited retinal degeneration. Science translational medicine 8(368):368rv366

17. Soleimannejad M, Ebrahimi-Barough S, Nadri S et al (2017) Retina tissue engineering by conjunctiva mesenchymal stem cells encapsulated in fibrin gel: Hypotheses on novel approach to retinal diseases treatment. Medical hypotheses 101:75-77

18. Mathew B, Poston JN, Dreixler JC et al (2017) Bone-marrow mesenchymal stem-cell administration significantly improves outcome after retinal ischemia in rats. Graefe's archive for clinical experimental ophthalmology = Albrecht von Graefes Archiv fur klinische experimentelle Ophthalmologie 255(8):1581-1592

19. Lumayag S, Haldin CE, Corbett NJ et al. Inactivation of the microRNA-183/96/182 cluster results in syndromic retinal degeneration. Proceedings of the National Academy of Sciences. 2013;110(6):E507-E516

20. Fan J, Jia L, Li Y et al. Maturation arrest in early postnatal sensory receptors by deletion of the miR183/96/182 cluster in mouse. Proceedings of the National Academy of Sciences. 2017:201619442

21. Furukawa T, Morrow EM, Cepko CL (1997) Crx, a novel otx-like homeobox gene, shows photoreceptorspecific expression and regulates photoreceptor differentiation. Cell 91(4):531-541

22. Koike C, Nishida A, Ueno $S$ et al (2007) Functional roles of Otx2 transcription factor in postnatal mouse retinal development. Molecular cellular biology 27(23):8318-8329

23. Tanimoto Y, Okada K, Hayashi F, Morigaki K (2015) Evaluating the Raftophilicity of Rhodopsin Photoreceptor in a Patterned Model Membrane. Biophysical journal 109(11):2307-2316

24. Pinzon-Guzman C, Zhang SS, Barnstable CJ (2011) Specific protein kinase $C$ isoforms are required for rod photoreceptor differentiation. The Journal of neuroscience: the official journal of the Society for Neuroscience 31(50):18606-18617

25. Lambrecht H-G, Koch K-W (1992) Recoverin, a novel calcium-binding protein from vertebrate photoreceptors. Biochimica et Biophysica Acta (BBA)-Protein Structure Molecular Enzymology 1160(1):63-66

26. Davari M, Soheili ZS, Samiei S, Sharifi Z, Pirmardan ER (2017) Overexpression of miR-183/-96/-182 triggers neuronal cell fate in Human Retinal Pigment Epithelial (hRPE) cells in culture. Biochemical biophysical research communications 483(1):745-751 
27. Komuta $\mathrm{Y}$, Ishii T, Kaneda $\mathrm{M}$ et al. In vitro transdifferentiation of human peripheral blood mononuclear cells to photoreceptor-like cells. Biology open. 2016:bio. 016477

28. Mellough CB, Sernagor E, Moreno-Gimeno I, Steel DH, Lako M (2012) Efficient stage-specific differentiation of human pluripotent stem cells toward retinal photoreceptor cells. Stem cells 30(4):673-686

\section{Figures}
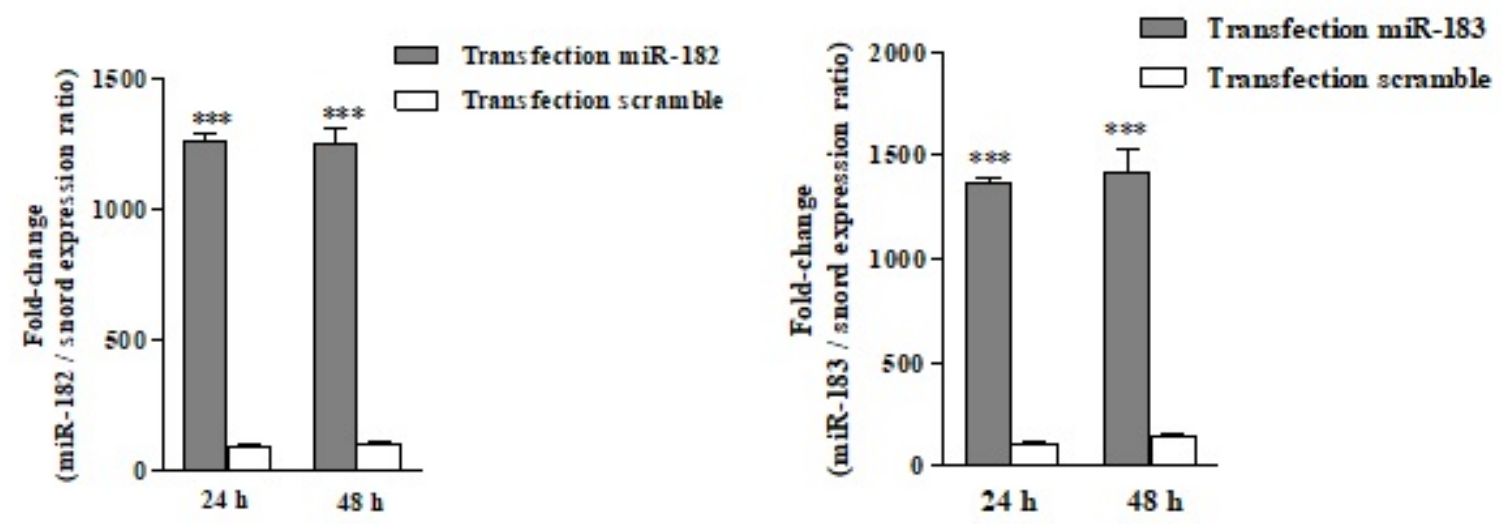

Figure 1

miRNA-182, -183 expression in hBMSCs transfected with miRNA-182, -183 and scramble. The qRT-PCR showed significant up-regulation of miRNA-182, -183 in transfected cells after 24 and 48 hours $(p<0.001)$. Asterisks showed significant expression rates: ${ }^{*} P<0.05$, ${ }^{\star *} P<0.001,{ }^{* \star * P}<0.001$

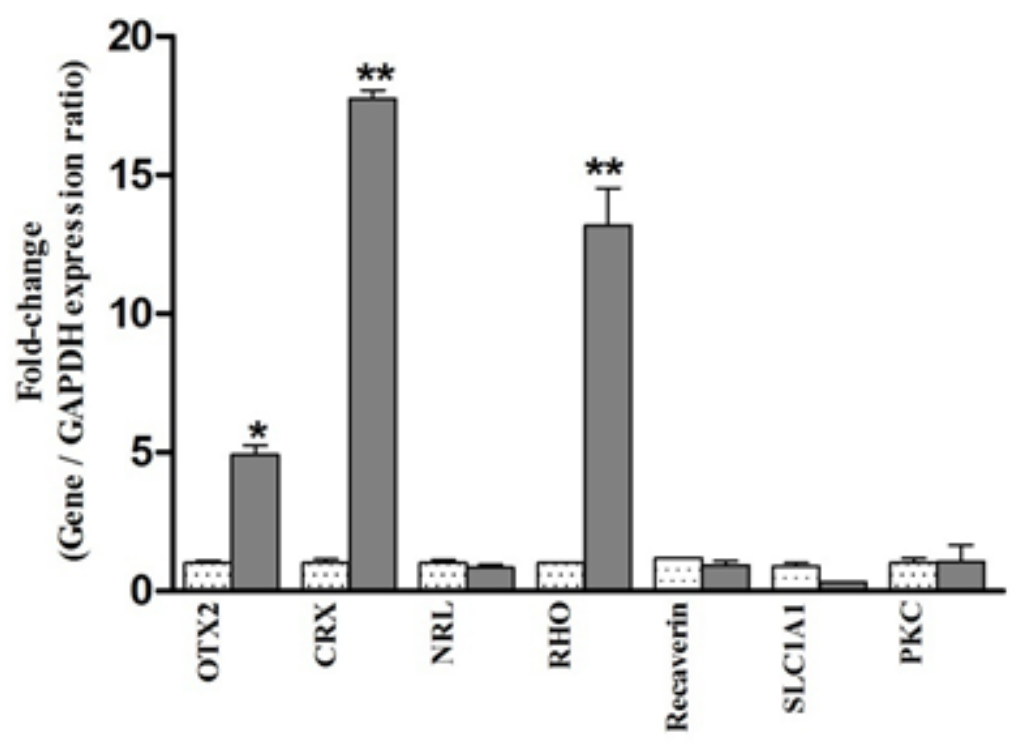

Figure 2 
Change in the expression of CRX, OTX2, PKC, NRL, SLC1A1, RHO and Recoverin genes compared with GAPDH gene after 24 hours in transfected cells. Results are expressed as fold change relative to the control group. Data is expressed as mean \pm SD.

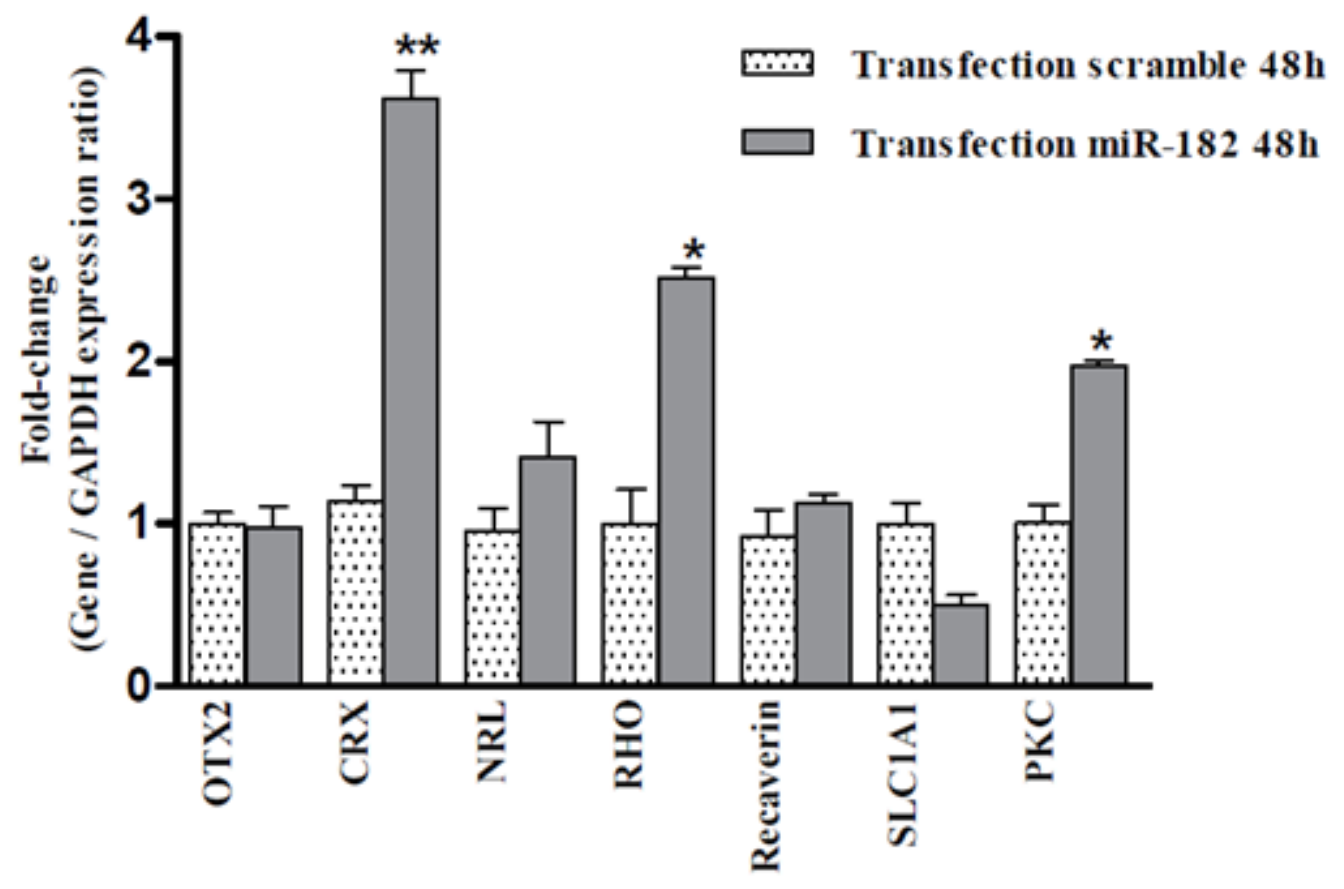

Figure 3

Changes in the expression of CRX, OTX2, PKC, NRL, SLC1A1, RHO and Recoverin genes compared with GAPDH gene after 48 hours in transfected cells. Results are expressed as fold change relative to the control group. Data is expressed as mean \pm SD.

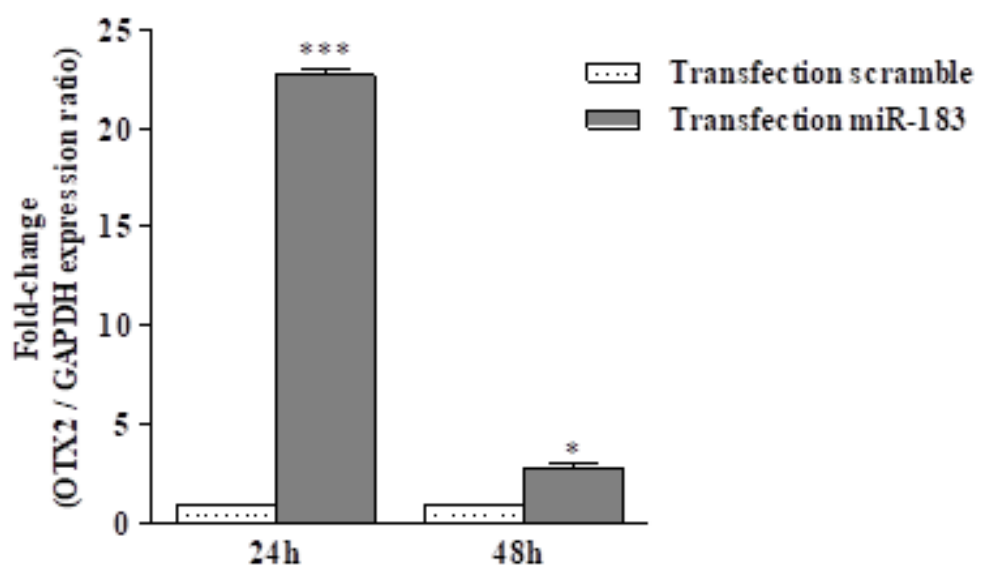

Figure 4

Changes in the expression of OTX2 gene compared with GAPDH gene after 24 and 48 hours in transfected cells. Results are expressed as fold change relative to the control group. Data is expressed as mean \pm SD. 


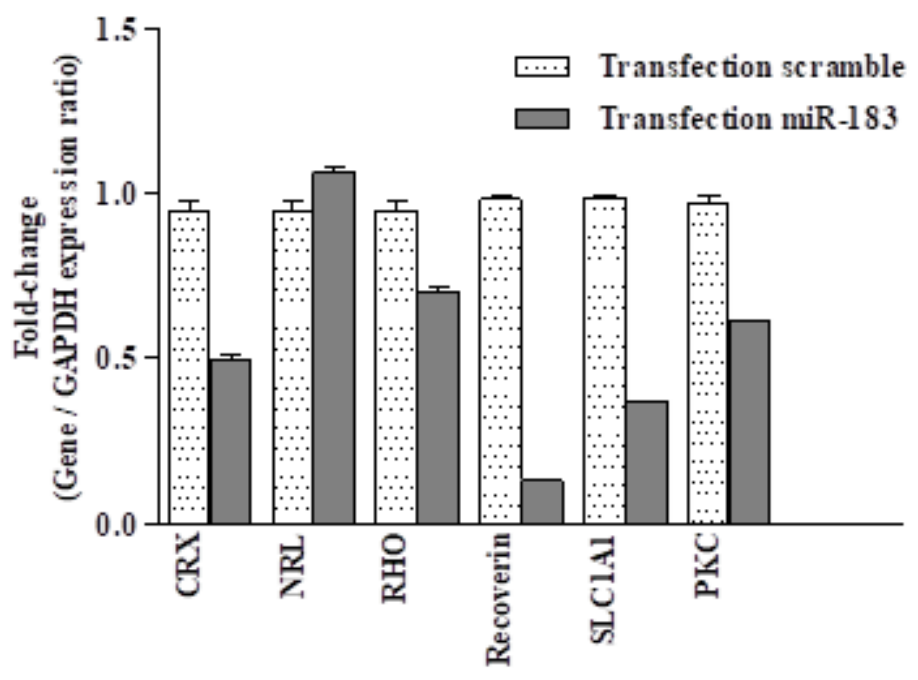

Figure 5

Changes in the expression of CRX, NRL, RHO, Recoverin, SLC1A1, and PKC genes compared with GAPDH gene after 24 hours in transfected cells. Results are expressed as fold change relative to the control group. Data is expressed as mean \pm SD.

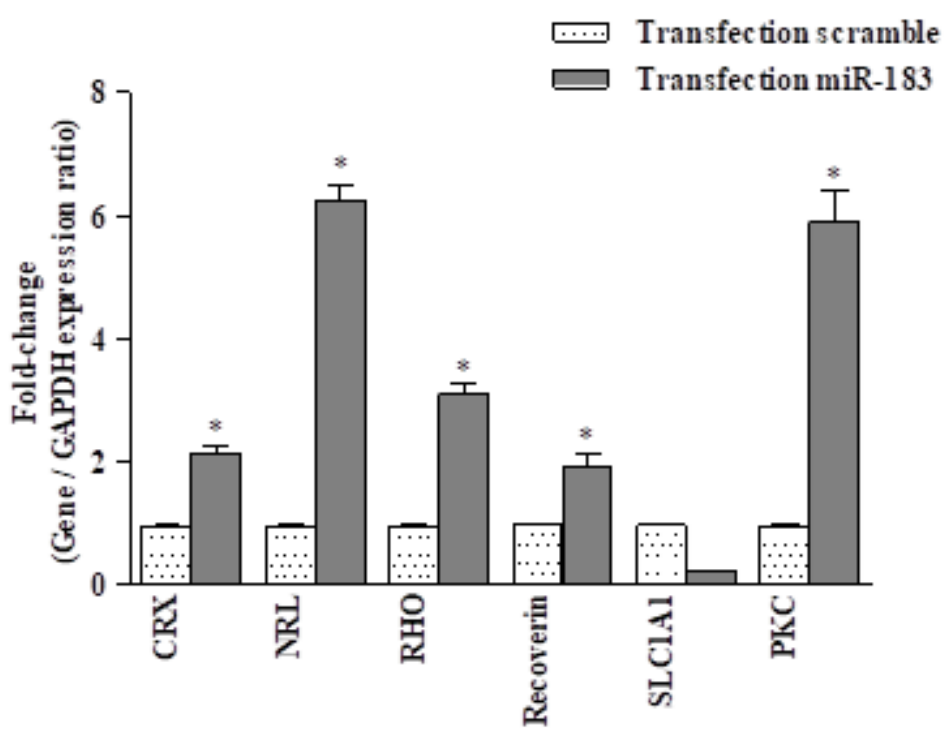

Figure 6

Changes in the expression of CRX, NRL, RHO, Recoverin, SLC1A1, and PKC genes compared with GAPDH gene after 48 hours in transfected cells. Results are expressed as fold change relative to the control group. Data is expressed as mean $\pm \mathrm{SD}$. 


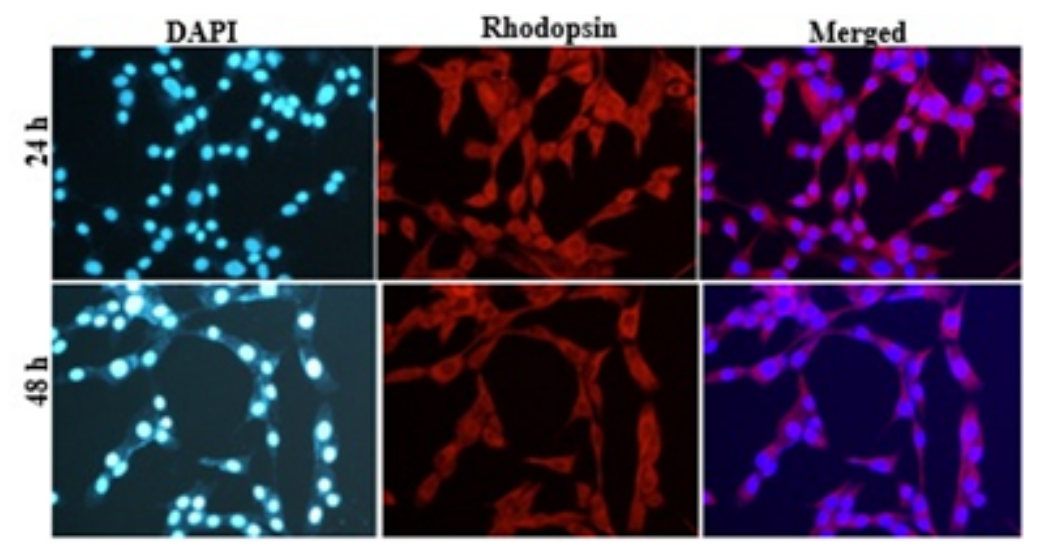

Figure 7

Immunofluorescence staining results of RHO 24 and 48 hours' after the transfection miR-182.

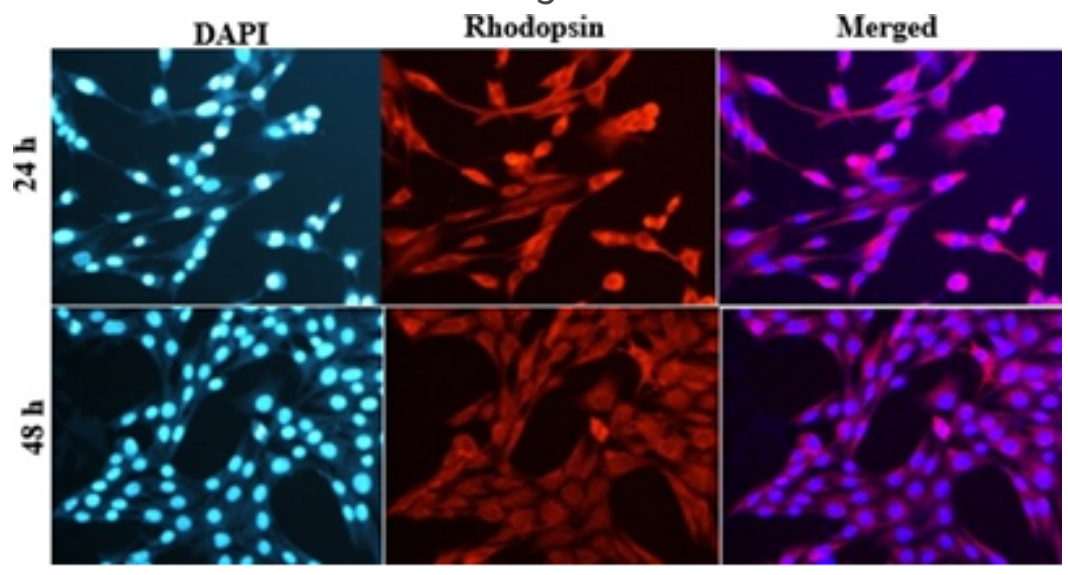

Figure 8

Immunofluorescence staining results of RHO 24 and 48 hours' after the transfection miR-183

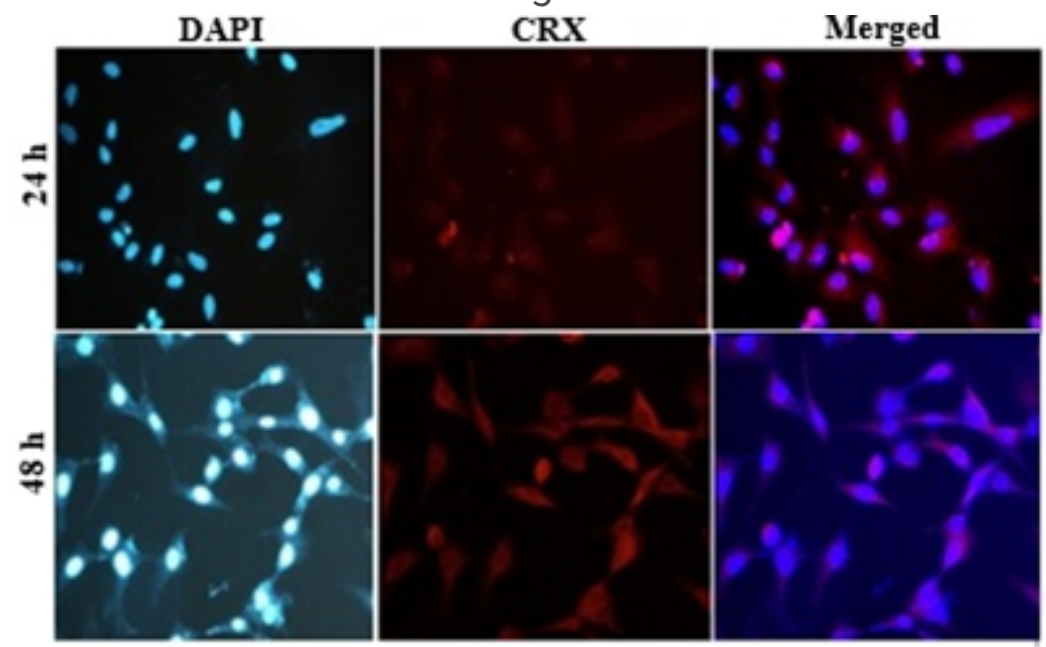

Figure 9

Immunofluorescence staining results of CRX 24 and 48 hours' after the transfection miR-182 


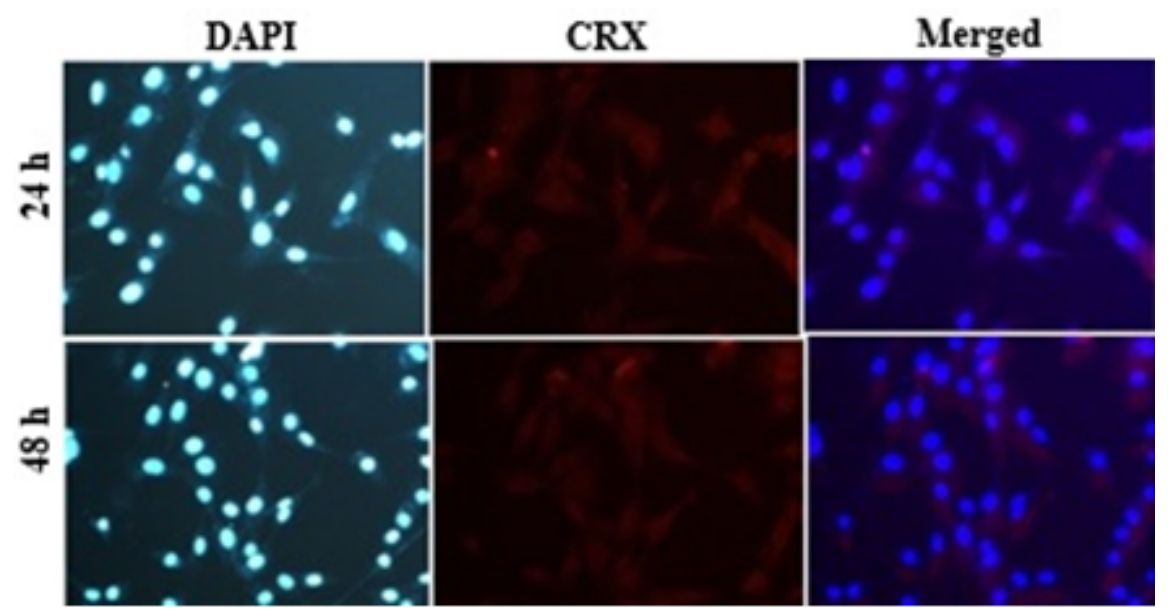

Figure 10

Immunofluorescence staining results of CRX 24 and 48 hours' after the transfection miR-183 